\title{
Mirrored Bilateral Slow-Wave Cortical Activity within Local Circuits Revealed by Fast Bihemispheric Voltage-Sensitive Dye Imaging in Anesthetized and Awake Mice
}

\author{
Majid H. Mohajerani, ${ }^{1,2}$ David A. McVea, ${ }^{1,2}$ Matthew Fingas, ${ }^{1,2}$ and Timothy H. Murphy ${ }^{1,2,3}$ \\ ${ }^{1}$ Kinsmen Laboratory, Department of Psychiatry, ${ }^{2}$ Brain Research Center, and ${ }^{3}$ Department of Cellular and Physiological Sciences, University of British \\ Columbia, Vancouver, British Columbia V6T 1Z3, Canada
}

\begin{abstract}
Spontaneous slow-wave oscillations of neuronal membrane potential occur about once every second in the rodent cortex and may serve to shape the efficacy of evoked neuronal responses and consolidate memory during sleep. However, whether these oscillations reflect the entrainment of all cortical regions via propagating waves or whether they exhibit regional and temporal heterogeneity that reflects processing in local cortical circuits is unknown. Using voltage-sensitive dye (VSD) imaging within an adult C57BL/6J mouse crossmidline large craniotomy preparation, we recorded this depolarizing activity across most of both cortical hemispheres simultaneously in both anesthetized and quiet awake animals. Spontaneous oscillations in the VSD signal were highly synchronized between hemispheres, and acallosal I/LnJ mice indicated that synchrony depended on the corpus callosum. In both anesthetized and awake mice (recovered from anesthesia), the oscillations were not necessarily global changes in activity state but were made up of complex local patterns characterized by multiple discrete peaks that were unevenly distributed across cortex. Although the local patterns of depolarizing activity were complex and changed over tens of milliseconds, they were faithfully mirrored in both hemispheres in mice with an intact corpus callosum, to perhaps ensure parallel modification of related circuits in both hemispheres. We conclude that within global rhythms of spontaneous activity are complex events that reflect orchestrated processing within local cortical circuits.
\end{abstract}

\section{Introduction}

Remarkably, much of the neural activity of the brain is unrelated to ongoing sensory or motor tasks. This spontaneous activity is characterized by rhythmic oscillations at frequencies ranging from infra-slow (under $0.1 \mathrm{~Hz}$ ) to ultrafast $(\sim 600 \mathrm{~Hz})$ (Buzsáki, 2006). Although the function of this activity is not yet understood, recent functional magnetic resonance imaging (fMRI) studies (Fox et al., 2006; Nir et al., 2008) have shown that infraslow-wave activity is characterized by synchronous bilateral activity in functionally related brain regions. These fMRI findings have suggested a role for infra-slow spontaneous activity in shaping or strengthening neural circuits (Nir et al., 2008) but cannot address the timescale of slow-wave oscillations and delta oscillations ranging from 0.3 to $4 \mathrm{~Hz}$. Slow waves occur during sleep and are composed of oscillations between depolarized ("up") and hyperpolarized ("down") membrane potential states (Timofeev et al., 2001; Destexhe and Contreras, 2006) and may underlie memory consolidation (Wilson and McNaughton, 1994; Steriade

\footnotetext{
Received Dec. 30, 2009; revised Jan. 23, 2010; accepted Jan. 26, 2010.

This work was supported by Canadian Institutes of Health Research (CIHR) Operating Grant MOP-12675 (T.H.M.), a Michael Smith Foundation for Health Research postdoctoral fellowship (M.H.M.), a (IHR Vanier Canada graduate scholarship (D.A.M.), and a Heart and Stroke Foundation of Canada and CIHR Focus on Stroke studentship (M.F.). We thank Pumin Wang and Cindy Jiang for assistance.

Correspondence should be addressed to Dr. Timothy H. Murphy, Department of Psychiatry, University of British Columbia, 4N1-2255 Wesbrook Mall, Vancouver, British Columbia V6T 1Z3, Canada. E-mail: thmurphy@interchange. ubc.ca.

DOI:10.1523/JNEUROSCI.6437-09.2010

Copyright $\odot 2010$ the authors $\quad 0270-6474 / 10 / 303745-07 \$ 15.00 / 0$
}

and Timofeev, 2003; Destexhe and Contreras, 2006) and shape responses to incoming stimuli in quiet awake or anesthetized rodents (Petersen et al., 2003; Waters and Helmchen, 2006). An important test of these predictions is to determine whether slowwave oscillations are regional dynamic features of brain as would be expected if they shape activity in select functional circuits (Kenet et al., 2003). Alternatively, slow waves may represent global or traveling wave-mediated changes in activity that entrain much of the cortex (Massimini et al., 2004). Multichannel EEG recordings have proven useful in addressing these hypotheses and have shown both local patterns of cortical up/down state (Sirota and Buzsáki, 2005) and widespread synchrony (Buzsaki et al., 1988) of cortical slow-wave activity. However, these approaches have been unable to resolve the regional structure and dynamics of these waves because of relatively sparse sampling. Previous studies (Prechtl et al., 1997; Huang et al., 2004; Ferezou et al., 2007) have shown voltage-sensitive dye (VSD) fluorescence to accurately reflect the spatial details of cortical membrane potential oscillations. Here, we expand these finding by imaging a region of cortex that encompasses much of the mouse neocortex with millisecond temporal resolution in awake and anesthetized animals. We find in both quiet awake and anesthetized animals that VSD signals indicate strong synchronization of spontaneous activity between hemispheres through transcallosal interhemispheric connections. However, close inspection revealed that these synchronized patterns were not global changes in cortical activity state (Massimini et al., 2004) but were composed of complex local patterns with several points of origin, indicating that 
they reflect the parallel engagement of select circuits within both hemispheres.

\section{Materials and Methods}

Animal model. Adult C57BL/6J and I/LnJ (The Jackson Laboratory) mice ( $>2$ months) were used. Animal protocols were approved by the University of British Columbia Animal Care Committee. Anesthesia was induced with urethane $(0.12 \% \mathrm{w} / \mathrm{w})$ or isoflurane $\left(0.5-2 \%\right.$ mixed in $\left.\mathrm{O}_{2}\right)$ for anesthetized and awake recording, respectively. Body temperature was maintained at $37 \pm 0.5^{\circ} \mathrm{C}$. A single cranial window was made over both mouse cortical hemispheres (either $6 \times 7 \mathrm{~mm}$; bregma 2 to $-4 \mathrm{~mm}$, lateral $0-3.5 \mathrm{~mm}$ or $7 \times 8 \mathrm{~mm}$; bregma 2.5 to $-4.5 \mathrm{~mm}$, lateral $0-4$ $\mathrm{mm})$. Lateral craniotomies were performed unilaterally $(7 \times 5 \mathrm{~mm}$; bregma 2.5 to $-4.5 \mathrm{~mm}$, lateral $0-5 \mathrm{~mm}$ ) on the right hemisphere.

VSD imaging. For in vivo VSD experiments, the dura was carefully removed within the craniotomy window. The dye, RH1692 (Optical Imaging) (Shoham et al., 1999), was dissolved in HEPES-buffered saline at an optical density of 5-7 (measured at $550 \mathrm{~nm}$ ) and applied to the exposed cortex for $90 \mathrm{~min}$. To minimize movement artifacts, the brain was covered with $1.5 \%$ agarose made in HEPES-buffered saline and sealed with a glass coverslip. VSD signals corresponded closely with cortical activity measured by surface EEG electrodes (supplemental Fig. $1 A-C$, available at www.jneurosci.org as supplemental material). Measurement of respiratory rhythm using a motion sensor indicated no contribution of breathing-induced hemodynamic signals or motion artifacts to VSD signals (supplemental Fig. $1 D$, available at www.jneurosci.org as supplemental material). For VSD data collection, 12-bit images were captured with 5.0 or 6.67 ms resolution with a digital camera (1M60 Pantera; Dalsa) and EPIX E1DB frame grabber with XCAP 3.1 imaging software (EPIX). VSD was excited with a red light-emitting diode (LED) (Luxeon $\mathrm{K} 2,627 \mathrm{~nm}$ ) as described previously (Brown et al., 2009). Images were taken through a macroscope composed of front-to-front video lenses $(8.4 \times 8.4 \mathrm{~mm}$ field of view, $65 \mu \mathrm{m}$ per pixel). To measure cortical activity evoked by sensory stimulation, we stimulated the hindlimbs and forelimbs using a piezoelectric device.

Recording behavior in awake animals during VSD imaging. Young (6-8 weeks old) male C57BL/6 mice $(n=5)$ were surgically implanted with a microconnector (CLP-106-02-FDH; Samtec USA) on the skull for immobilization by connecting to a fixed connector. After $4 \mathrm{~d}$ of recovery, mice were habituated to head restraint as described previously (Poulet and Petersen, 2008). Once habituated, a $7 \times 8 \mathrm{~mm}$ craniotomy was performed under isoflurane anesthesia $(0.5-2 \%$ maintenance mixed with oxygen) and then transferred to an awake imaging setup in which they were head restrained in a relaxed posture, and, when in training, they were given food pellets placed on a platform. To wake the animals, the isoflurane and oxygen were stopped, the anesthesia mask was removed, and VSD imaging data was obtained over the next $2-3 \mathrm{~h}$. Awake VSD imaging of spontaneous activity imaging was performed in the absence of visual, olfactory, or auditory stimulation during $30 \mathrm{~s}$ epochs. The $627 \mathrm{~nm}$ LED light used for VSD excitation or the infrared light used for behavioral observation did not result in a visual response. Data from the first second of awake imaging was discarded as a precaution against visual stimulation. The use of two behavioral monitoring cameras confirmed that the animals were indeed awake and relatively unstressed since grooming, whisking, feeding, and drinking were observed. An analgesic, bupremorphine, was injected $(0.075 \mathrm{mg} / \mathrm{kg}$, i.p.) $8 \mathrm{~h}$ before awake VSD recording. We used the second Dalsa $1 \mathrm{M} 60$ camera $(75$ or $150 \mathrm{~Hz})$ to capture whisker movements under infrared illumination.

Data analysis. VSD responses to sensory-evoked stimulation were calculated as the normalized difference to the average baseline recorded before stimulation $\left(\Delta F / F_{0} \times 100\right)$ using NIH ImageJ with a customwritten plugin (Harrison et al., 2009). To measure spontaneous activity, the images were collected for 10-30 s epochs with 5 or $6.67 \mathrm{~ms}$ temporal resolution and analyzed offline using custom-written code in Matlab and NIH ImageJ. Slow, time-dependent reductions in fluorescence were corrected using a Butterworth high-pass filter (zero-phase filter) at $0.5 \mathrm{~Hz}$. The fluorescence changes were quantified as $\left(F-F_{0}\right) / F_{0} \times 100$, where $F$ represents the fluorescence signal at any given time, and $F_{0}$ represents the average of fluorescence over all frames.
To measure the interhemispheric correlation map, both cortical hemispheres were divided into 10 regions (shown in Fig. $1 \mathrm{~A}$ ), including the following: (1) anterior cingulate (dorsal part) and anterior segment of secondary motor cortex, (2) forelimb primary somatosensory cortex, (3) anterior cingulate (dorsal and ventral part) and posterior segment of secondary motor cortex, (4) hindlimb somatosensory cortex, (5) C2 primary barrel cortex, (6) parietal association cortex, (7) retrosplenial cortex, (8) primary visual cortex, (9) secondary visual cortex, and (10) primary motor cortex. All cortical regions were identified based on stereotaxic coordinates and their stereotyped position relative to the functionally defined hindlimb area. In most experiments, the functional locations of the left and right forelimb and C2 whisker barrel maps were confirmed using evoked VSD responses. The extent of correlation between cortical regions was measured as described previously (Lampl et al., 1999).

EEG recording. A Teflon-coated chlorided silver wire $(0.125 \mathrm{~mm})$ was placed on the cortical surface. A reference electrode was placed on the nasal bone. The cortical signal was amplified and filtered $(0.1-1000 \mathrm{~Hz})$ using a differential alternating current amplifier.

Tracer injections and histology. Two I/LnJ mice were injected with the neuronal tracer cholera toxin subunit B $(1 \%)$ as described previously (Brown et al., 2009).

Statistical analysis. One-way ANOVA, adjusted using Tukey's method, was used to compare the frequency of spontaneous activity across different cortical regions. All $p$ values $<0.05$ were considered statistically significant.

\section{Results}

Spontaneous VSD signals were measured with 5-6.7 ms resolution over most of the neocortex simultaneously by imaging VSD signals that reflect changes in membrane potential (Shoham et al., 1999; Grinvald and Hildesheim, 2004; Ferezou et al., 2007) (Fig. 1A-C) (supplemental Movie 1, available at www.jneurosci. org as supplemental material). We began by recording simultaneously from primary (motor, somatosensory, visual), secondary (motor, visual), and association (retrosplenial, cingulate) cortices of both brain hemispheres of anesthetized mice (supplemental Fig. 2A,B shows stained region in detail, available at www. jneurosci.org as supplemental material). A striking observation was that VSD signals in both hemispheres were strongly correlated in both time and space. Figure $1, C$ and $D$, shows a representative VSD signal under urethane anesthesia that moves across both hemispheres in a similar pattern. Taking advantage of the large region of cortex from which we recorded, we created a catalog of correlation by selecting 10 regions of interest (Fig. 1A) and calculating the correlation coefficient between VSD signal fluctuations in the two hemispheres in each of these regions. Figure $1 D$ shows that bilateral activity in all regions of interest was correlated, with strongest interhemispheric correlation between medially located association cortices such as the motor cortex, anterior cingulate, and retrosplenial cortex. These cortical areas are located at the main hub of the connectional core of the brain and have been suggested by other methods to be part of a default network (Alkire et al., 2008; Hagmann et al., 2008). As expected, correlation coefficients between signals artificially separated in time (1-10 s) was very low $(r<0.1, n=8$ mice).

A reasonable hypothesis arising from these findings is that the corpus callosum is involved in synchronizing bilateral activity. To test this, we examined the correlation of interhemispheric spontaneous activity in acallosal mice (I/LnJ; The Jackson Laboratory). Without the interhemispheric connections carried in the corpus callosum and severe deficiency of the hippocampal commissure (Livy and Wahlsten, 1991) (as shown in supplemental Fig. $2 E, F$, available at www.jneurosci.org as supplemental material), spontaneous activity was less synchronized between hemi- 
A

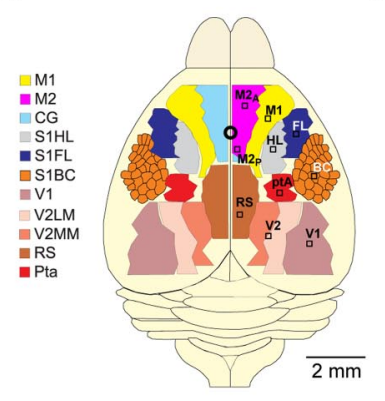

B

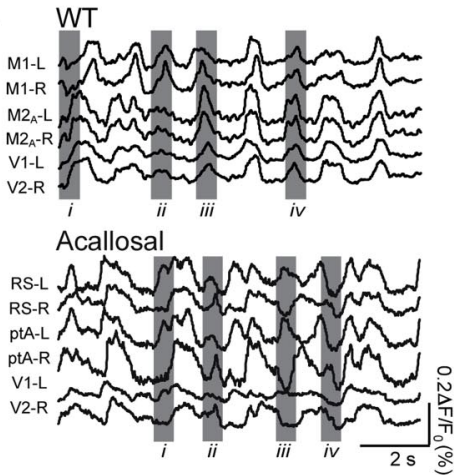

C

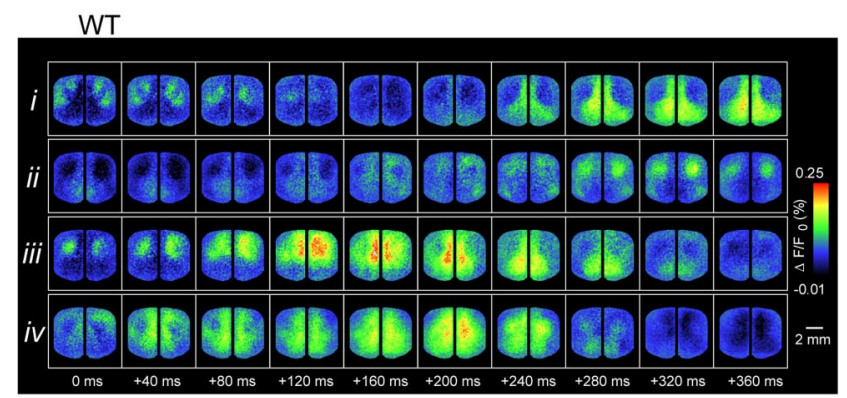

Acallosal

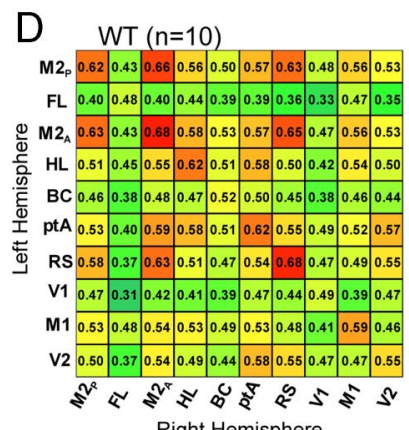

Right Hemisphere

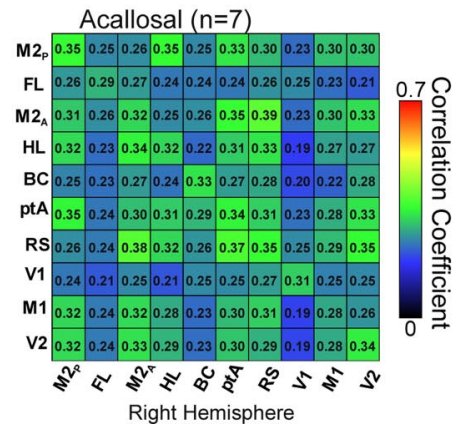

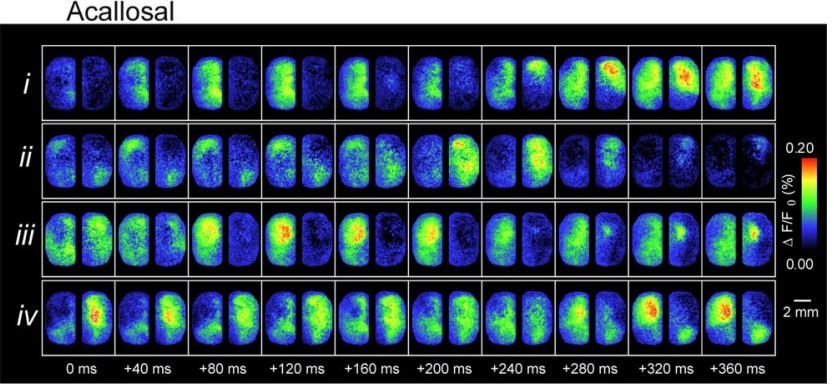

Figure 1. VSD imaging reveals spontaneous fluctuations of cortical activity are regionally heterogeneous and bilaterally synchronous in wild-type but not acallosal I/LnJ mice. $A$, Schematic showing mouse cortical regions imaged. Secondary motor cortex is removed in left hemisphere to show anterior cingulate (beneath it), found in the medial wall of the cortex in the longitudinal fissure. Note that signals from these two regions cannot be separately resolved. $\boldsymbol{B}$, Example traces showing VSD signal from selected regions in wild-type (WT) and acallosal I/LnJ mice. $\boldsymbol{C}$, Montages showing VSD signal from entire imaging field. $\boldsymbol{i}$-iv correspond to grayed regions of traces in $\boldsymbol{B}$. $\boldsymbol{D}$, Matrices showing pairwise correlation coefficients for interhemispheric regions (shown in $\boldsymbol{A}$ ) in wild-type and acallosal I/LnJ mice. BC, C2 primary barrel cortex; CG, cingulate cortex; FL and S1FL, forelimb primary somatosensory cortex; HL and S1HL, hindlimb primary somatosensory cortex; M1, primary motor cortex; $\mathrm{ML}_{\mathrm{A}}$, anterior cingulate (dorsal part) and anterior segment of secondary motor cortex; $\mathrm{M} 2 \mathrm{p}$, anterior cingulate (dorsal and ventral part) and posterior segment of secondary motor cortex; ptA, parietal association cortex; RS, retrosplenial cortex; S1BC, primary somatosensory barrel cortex; V1, primary visual cortex; V2, secondary visual cortex; V2LM, lateromedial secondary visual cortex; V2MM, mediomedial secondary visual cortex; L, left; $R$, right. The brain is oriented so that the anterior direction is up and both hemispheres are shown.

spheres (Fig. $1 B-D)$ (supplemental Movie 2, available at www. jneurosci.org as supplemental material), although the frequency distribution of the activity was not changed (supplemental Fig. 3, available at www.jneurosci.org as supplemental material). It is likely that subcortical structures such as the thalamus and hippocampus, which have strong influences on synchronous cortical activity (Timofeev and Steriade, 1996; Hoffman et al., 2007), contribute to the residual correlation in $\mathrm{I} / \mathrm{LnJ}$ mice.

In awake rodents engaged in specific tasks such as whisking, cortical activity is relatively desynchronized (Poulet and Petersen, 2008). In contrast, cortical neurons of quiet awake rodents exhibit membrane potential oscillations (Crochet and Petersen, 2006) that are locally synchronized and mirror low frequency (3-6 Hz) EEG oscillations. Whether this synchrony in quiet awake periods extends across wide regions of the cortex and between hemispheres is unknown. To examine this question, we recorded bilateral cortical activity in awake mice with minimal sensory input (for details, see Materials and Methods). Overall synchrony, as revealed by EEG, varied with behavioral state, consistent with the previous work described above. EEG activity exhibited higher frequencies during behavior than anesthesia or quiet wakefulness (Fig. $2 B$ ) (supplemental Fig. $4 A, B$, available at www.jneurosci.org as supplemental material). Using VSD signals, we show in quiet awake animals that discrete cortical regions were synchronized bilaterally and that the pattern of correlation between corresponding interhemispheric regions was similar to those observed while under anesthesia (Fig. 2A,C) (supplemental Movie 3, available at www.jneurosci.org as supplemental material). It is unlikely that such patterns are an aftereffect of anesthesia because there was no significant difference between bihemispheric correlation 30 or 90 min after the cessation of isoflurane ( $n=6$ mice, $p=0.65$ ). This suggests that features of the nervous system that lead to synchronized membrane potential oscillations in anesthetized animals are also active in quiet awake animals.

While examining bilateral activity (in both awake and anesthetized animals), we noted that oscillations rarely moved in a single coherent direction. For example, Figure $3 A$ shows an oscillation that moves in a spiral: first posteriorly, then medially, and then returns to an isolated anterior region, indicating a dependence on underlying local circuit structure (see also supplemental Movies 1, 4, available at www.jneurosci.org as supplemental material). Such patterns are inconsistent with the current understanding of slow-wave oscillations as traveling waves of cortical depolarization originating from a single point of origin or reflecting changes in global cortical state (Massimini et al., 2004). These findings motivated us to explore the movement of oscillations over larger lateral distances, and we shifted our imaging region to a single hemisphere to include the auditory, secondary somatosensory, and lateral secondary visual cortices (supplemental Fig. $2 C, D$, shows stained region, available at www.jneurosci.org as supplemental material). Figure $3 A$ shows two example oscillations seen from this wider cranial window. In both cases, VSD signal varies widely across different regions and over time, although associated surface EEG suggests a single depolarization (supplemental Movie 4, available at www.jneurosci.org as supplemental material). Spatial heterogeneity in membrane potential changes across the cortex was a consistent feature of 


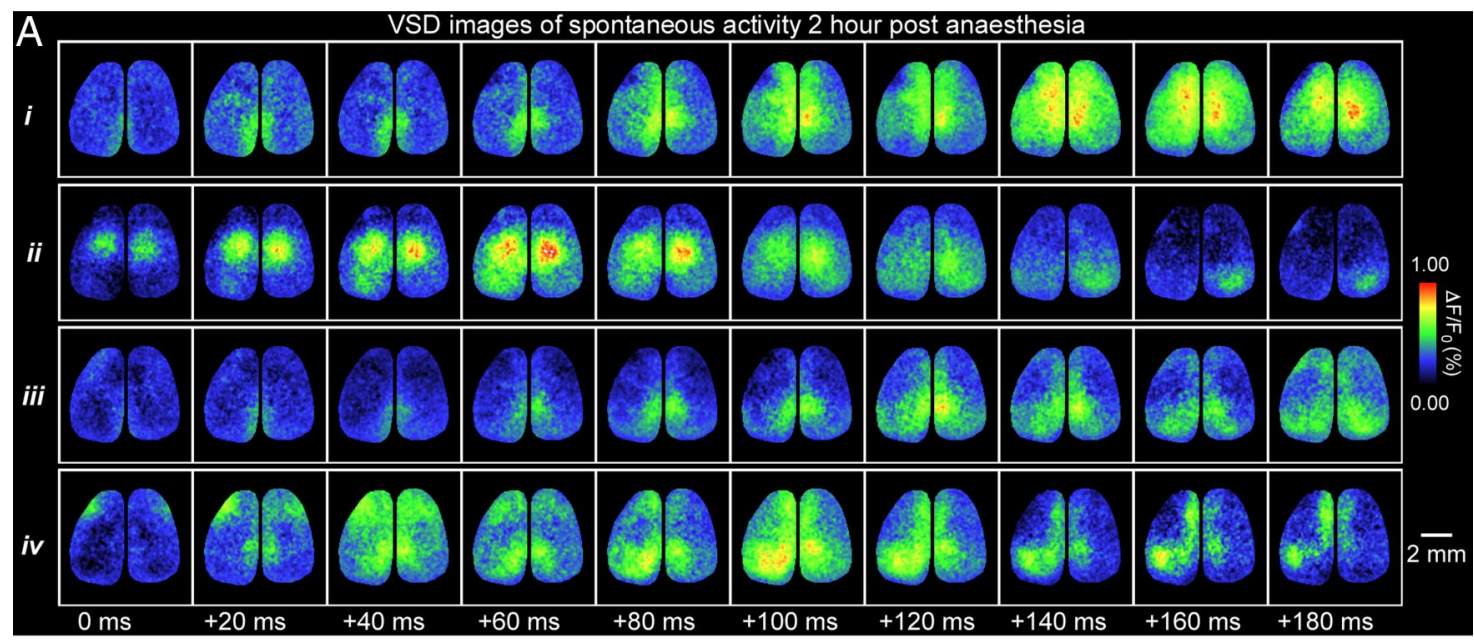

B

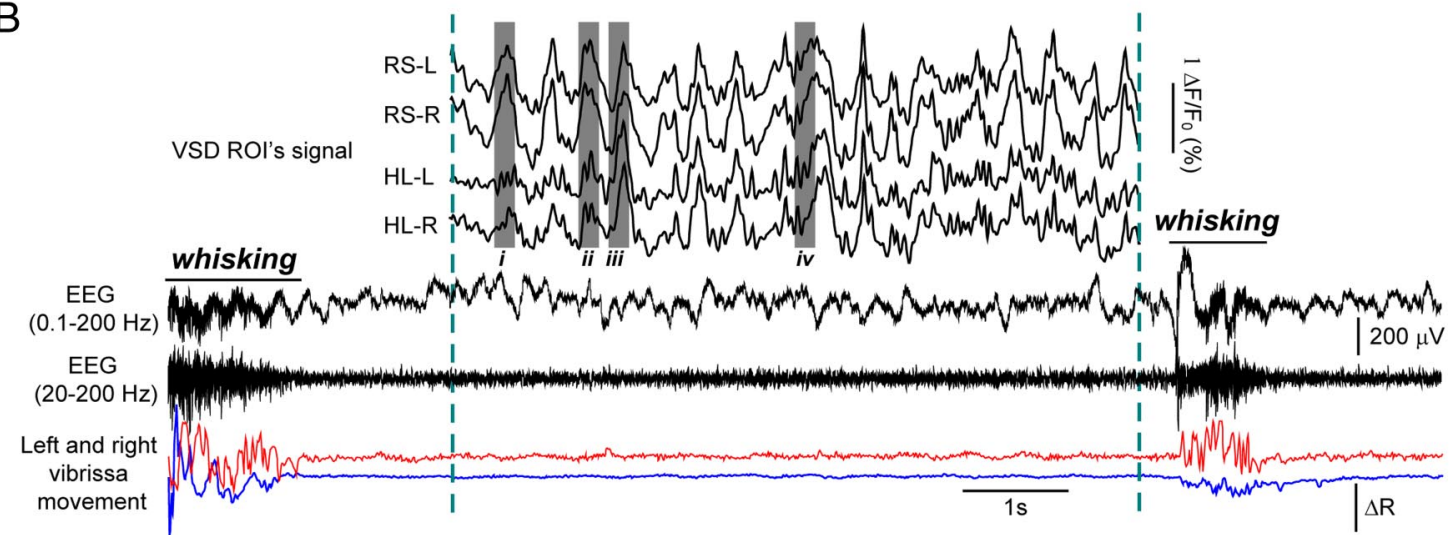

C

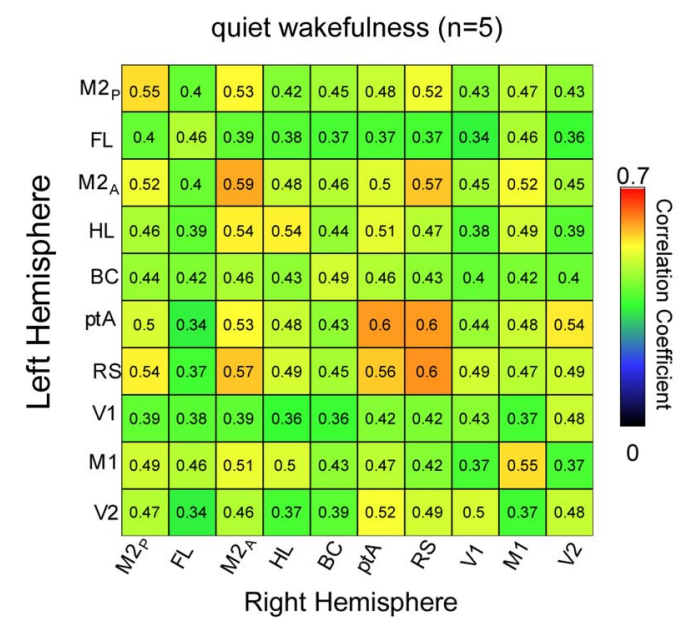

Anesthetized correlation/ awake correlation \begin{tabular}{l|l|l|l|l|l|l|l|l|l|l|}
\hline M2 & 0.88 & 0.92 & 0.81 & 0.75 & 0.90 & 0.84 & 0.83 & 0.89 & 0.84 & 0.82 \\
\hline
\end{tabular}

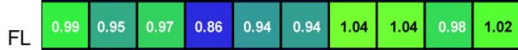

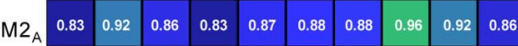

\begin{tabular}{ll|l|l|l|l|l|l|l|l|l}
$\mathrm{HL}$ & 0.90 & 0.87 & 0.98 & 0.87 & 0.87 & 0.88 & 0.94 & 0.90 & 0.91 & 0.79
\end{tabular}

\begin{tabular}{ll|l|l|l|l|l|l|l|l|l|}
\hline BC & 0.96 & 1.11 & 0.95 & 0.91 & 0.94 & 0.93 & 0.95 & 1.04 & 0.91 & 0.91 \\
\hline
\end{tabular}

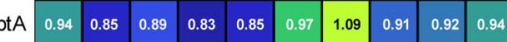

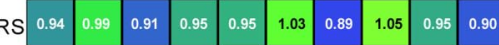

\begin{tabular}{lllllllllllll} 
V1 & 0.83 & 1.21 & 0.92 & 0.88 & 0.91 & 0.90 & 0.95 & 0.87 & 0.95 & 1.03 \\
\hline
\end{tabular}

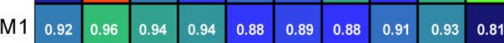

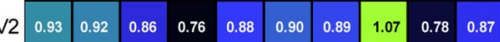

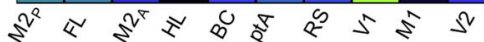

Right Hemisphere

Figure 2. Spontaneous activity during quiet wakefulness is also bilaterally synchronous. $A$, Montage showing four sequences of VSD signal from both hemispheres of the cortex of a quiet awake mouse. $\boldsymbol{i}$-iv correspond to grayed regions of traces in $\boldsymbol{B}$. $\boldsymbol{B}$, Example traces (i) showing VSD signal from regions (see Fig. $1 \mathrm{~A}$ ) of an awake head-restrained mouse $2 \mathrm{~h}$ after stopping gas anesthesia and 1.5 s after an episode of whisking. ii, EEG signals filtered between $0.1-200 \mathrm{~Hz}$ (top trace) and $20-200 \mathrm{~Hz}$ (bottom trace). iii, Left (blue) and right (red) vibrissae movements monitored by video and changes in reflected light. During quiescence, the EEG shows low-frequency activity (ii, bottom trace), whereas during whisking, higher frequency fluctuations are observed. ROI, Region of interest. C,Matrix showing pairwise correlation coefficients for interhemispheric regions from $n=5$ awake animals. $\boldsymbol{D}$, Correlation coefficient matrix for wild-type anesthetized mice/wild-type quiet awake mice, indicating similar degrees of correlation. The brain is oriented so that the anterior direction is up.

depolarizing events; single peak depolarizations were rare (Fig. $3 B, C$ ), and depolarizations often originated from multiple cortical regions within a single $6.7 \mathrm{~ms}$ frame (Fig. $3 A$ ). This heterogeneity was reflected in the overall activity of these brain regions, because mean frequency of depolarization was significantly higher in association cortices than primary sensory cortices (Fig. 3D; see also Fig. $1 B$ ) (supplemental Movie 1, available at www.jneurosci.org as supplemental material).

\section{Discussion}

Here we monitor VSD signals that report changes in membrane potential over large regions of the mouse cortex to study the 

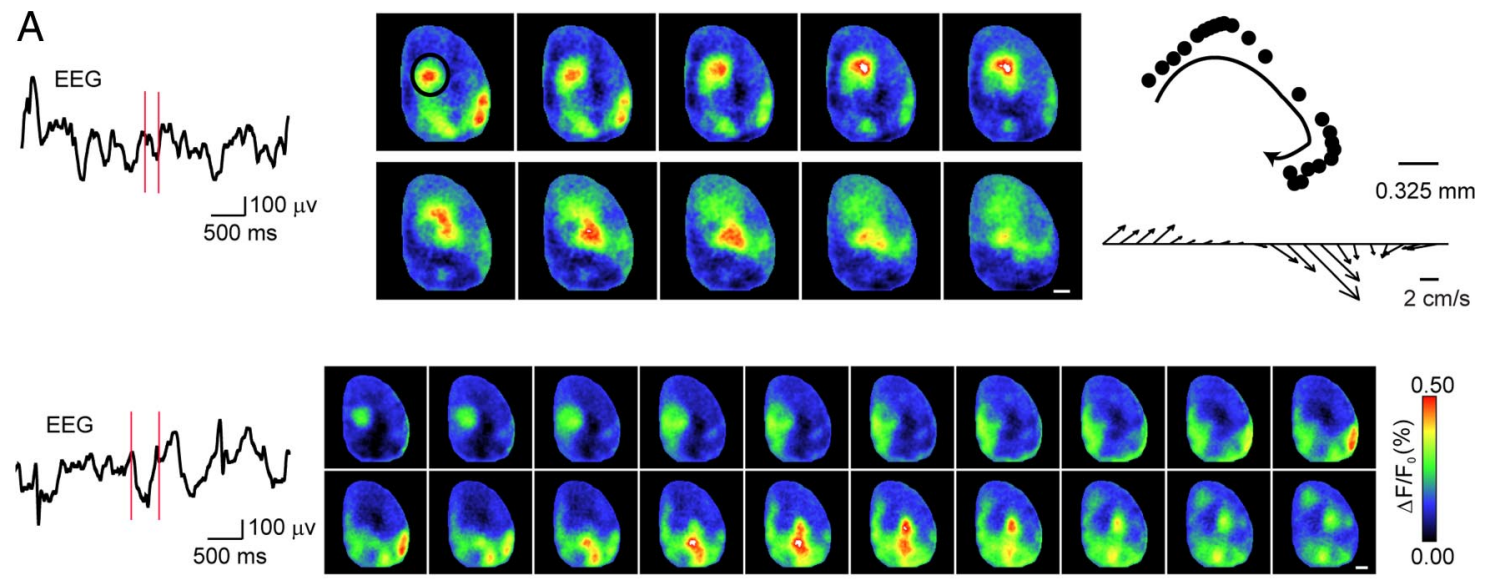

B

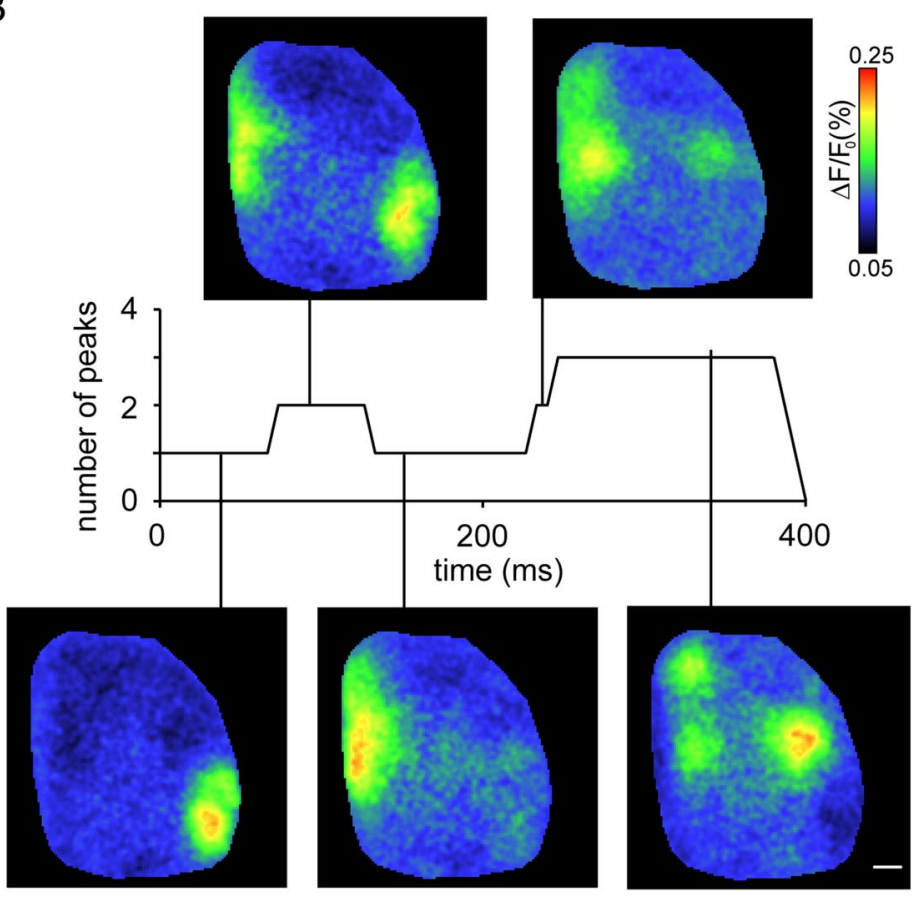

C

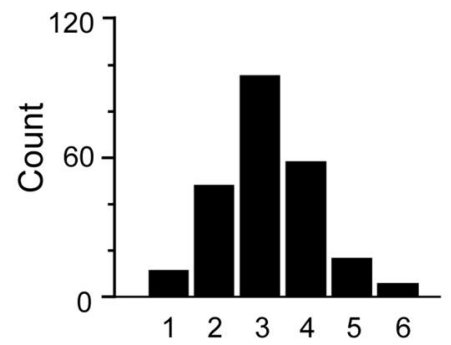

Maximum number of peaks

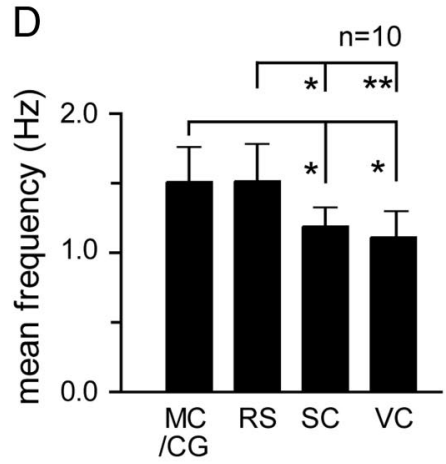

Cortical region

Figure 3. Large unilateral imaging region (within the right hemisphere) reveals that slow-wave activity is composed of complex local events. A, Two examples of complex patterns of cortical membrane potential oscillations. Surface EEG is displayed on the left, and images corresponding to the region between the two red vertical markers are shown; images were taken every $13.4 \mathrm{~ms}$ for the upper example and every $20 \mathrm{~ms}$ for the lower example. Top at right shows spiral path and instantaneous velocity of a single peak (in black circle, first panel). Scale bar, $1 \mathrm{~mm}$. $\boldsymbol{B}$, Single example showing that the number of discrete peaks of cortical depolarization evolves rapidly over time as activity spreads across the cortex. Vertical lines connect VSD images to the appropriate point on the record above that provides an index of the number of peaks detected. Scale bar, $1 \mathrm{~mm}$. C, Histogram showing the number of discrete cortical depolarizations during slow-wave events. D, Bar graph showing mean frequency of local depolarization for four cortical regions: $M C / C G$, motor cortex/anterior cingulate; $R S$, retrosplenial cortex; $S C$, somatosensory cortex; VC, primary visual cortex. Error bars show $1 \mathrm{SE}_{;} ;{ }^{*} p<0.05 ;{ }^{* *} p<0.01$. The brain is oriented so that the anterior direction is up and the lateral edge toward the right.

spatial and temporal dynamics of spontaneous cortical oscillations present in the anesthetized and quiet awake mouse brain. This activity is highly synchronized across multiple brain regions and both hemispheres and, in particular, association cortices located in midline regions. Previous studies, using fMRI to measure much slower brain oscillations (infra-slow oscillations $<0.1 \mathrm{~Hz}$ ) have shown a similar regional pattern (Fox et al., 2006). This pattern may reflect a default network of regions that are active during rest and tasked with global monitoring of internal environment and multimodal integration of information (Alkire et al., 2008). Our results are generally consistent with this hypothesis and now extend the observation to significantly higher frequencies. As in infra-slow activity in the human and primate brain, higher-frequency slow-wave oscillation in the mouse brain was most highly correlated and frequent near the midline in the anterior cingulate and retrosplenial cortex, implicating these regions as the major hub of information processing during spontaneous activity.

The second key finding is the presence of unique patterns of depolarizing activity that are restricted to local regions of cortical circuits yet broadcast bilaterally. Although multichannel EEG can detect gross regional differences in the distribution of slow-wave sleep activity (Huber et al., 2008), the local features we describe could be hidden from traditional recording techniques and emphasize the need to record both subthreshold and suprathreshold activity (reported by VSD imaging) from wide regions of the 
cortex relatively non-invasively with high temporal resolution (Ferezou et al., 2007). Here the relatively small mouse cortex that lacks the complexity of a gyrencephalic (primate or feline) brain provides the ideal platform to image such events. The benefit of such widespread recordings is emphasized by the decrease in synchrony of intrahemispheric VSD signals that are found as the distance between functional regions is increased. Importantly, although spatial heterogeneity was observed over relatively large distances between lateral regions and near midline, local signals recorded within a functional domain were more synchronous, consistent with previous work in cat (Destexhe et al., 1999) and our data within the supplemental Movie 1 legend (available at www.jneurosci.org as supplemental material). Although regional intrahemispheric differences in synchrony were apparent, even regions at the largest distances apart (that were examined), such as motor cortex and primary visual cortex, were still significantly correlated, albeit more weakly ( $\sim 6 \mathrm{~mm}$ apart in mouse; $r=0.38$, $n=10, p<0.05)$ during slow-wave activity, consistent with synchrony observed over similar distances in cat. The existence of similar activity during quiet wakefulness also reinforces the value of such recordings and strengthens the hypothesis that ongoing activity in select circuits shapes the response of the brain during the transition from quiescence to active processing of sensory inputs (Petersen et al., 2003).

Slow-wave and delta oscillations are generated through both intracortical and to a lesser extent corticothalamic activity (Steriade and Timofeev, 2003). Our results suggest that synchronization between cortical hemispheres is mediated to a large part by the corpus callosum. Perhaps during spontaneous slow-wave rhythms (in contrast to sensory-evoked activation), transcallosal connections are relatively disinhibited, favoring projections to the opposite homotypic cortex. Consistent with this proposal, mirrored transcallosal spread of activity was not observed during sensory-evoked activity when assayed by VSD imaging (Ferezou et al., 2007; Brown et al., 2009). Regarding noncortical influences on synchrony, although spatial interhemispheric correlations were greatly reduced in acallosal mice, there was still a low level of temporal correlation between cortical hemispheres. Residual bilateral temporal synchrony that remains in acallosal mice may arise from interconnected subcortical regions, such as the anterior commissure, which are not significantly different from wild-type mice (Livy and Wahlsten, 1991) or interhemispheric thalamic connections (Timofeev and Steriade, 1996). The hippocampal commissure is unlikely to contribute because it is shrunken compared with wild-type mice, although not to the same extent as the corpus callosum (Livy and Wahlsten, 1991). Recording from, or inactivating, thalamic or other subcortical structures is a useful technique to address these issues and may shed light on the mechanism of synchronizing cortical oscillations. Buzsaki et al. (1988), for example, showed that unilateral lesion of the thalamus did not change power spectrum in the 1-4 $\mathrm{Hz}$ band of EEG activity. Nonetheless, a clear mechanistic account from this approach is unlikely because these oscillations reflect a complex system in which cortical and subcortical areas are likely cooperatively involved through reciprocal connections (Steriade and Timofeev, 2003; Sirota and Buzsáki, 2005).

Our results agree with the general idea that, during slow-wave activity, there are widespread shifts of membrane potential (Steriade et al., 1993). However, we now draw attention to local oscillations that are superimposed on slow waves. Remarkably, we find that the spatial details of these local oscillations are reproduced with high fidelity in both hemispheres and are relevant to both awake and anesthetized states. Precise interhemispheric spatial correla- tion of local neuronal activity during quiescence or sleep may serve to ensure the parallel modification of functionally related synaptic connections in both hemispheres. Furthermore, the fact that these complex patterns of slow-wave activity are mirrored in both hemispheres to within tens of milliseconds suggests that they are a fundamental property of cortical dynamics, although exceptions exist in cetaceans (Mukhametov et al., 1977). Conceivably, processes in which slow-wave activity are implicated, including sensory perception, motor integration, and memory consolidation during sleep (Huber et al., 2008; Haider and McCormick, 2009), will need to consider that slow-wave activity reflects local cortical circuit processing events that are broadcast bilaterally with high fidelity.

\section{References}

Alkire MT, Hudetz AG, Tononi G (2008) Consciousness and anesthesia. Science 322:876-880.

Brown CE, Aminoltejari K, Erb H, Winship IR, Murphy TH (2009) In vivo voltage-sensitive dye imaging in adult mice reveals that somatosensory maps lost to stroke are replaced over weeks by new structural and functional circuits with prolonged modes of activation within both the periinfarct zone and distant sites. J Neurosci 29:1719-1734.

Buzsaki G (2006) Rhythms of the brain. Oxford, UK: Oxford UP.

Buzsaki G, Bickford RG, Ponomareff G, Thal LJ, Mandel R, Gage FH (1988) Nucleus basalis and thalamic control of neocortical activity in the freely moving rat. J Neurosci 8:4007-4026.

Crochet S, Petersen CC (2006) Correlating whisker behavior with membrane potential in barrel cortex of awake mice. Nat Neurosci 9:608-610.

Destexhe A, Contreras D (2006) Neuronal computations with stochastic network states. Science 314:85-90.

Destexhe A, Contreras D, Steriade M (1999) Spatiotemporal analysis of local field potentials and unit discharges in cat cerebral cortex during natural wake and sleep states. J Neurosci 19:4595-4608.

Ferezou I, Haiss F, Gentet LJ, Aronoff R, Weber B, Petersen CC (2007) Spatiotemporal dynamics of cortical sensorimotor integration in behaving mice. Neuron 56:907-923.

Fox MD, Corbetta M, Snyder AZ, Vincent JL, Raichle ME (2006) Spontaneous neuronal activity distinguishes human dorsal and ventral attention systems. Proc Natl Acad Sci U S A 103:10046-10051.

Grinvald A, Hildesheim R (2004) VSDI: a new era in functional imaging of cortical dynamics. Nat Rev Neurosci 5:874-885.

Hagmann P, Cammoun L, Gigandet X, Meuli R, Honey CJ, Wedeen VJ, Sporns O (2008) Mapping the structural core of human cerebral cortex. PLoS Biol 6:e159.

Haider B, McCormick DA (2009) Rapid neocortical dynamics: cellular and network mechanisms. Neuron 62:171-189.

Harrison TC, Sigler A, Murphy TH (2009) Simple and cost-effective hardware and software for functional brain mapping using intrinsic optical signal imaging. J Neurosci Methods 182:211-218.

Hoffman KL, Battaglia FP, Harris K, MacLean JN, Marshall L, Mehta MR (2007) The upshot of up states in the neocortex: from slow oscillations to memory formation. J Neurosci 27:11838-11841.

Huang X, Troy WC, Yang Q, Ma H, Laing CR, Schiff SJ, Wu JY (2004) Spiral waves in disinhibited mammalian neocortex. J Neurosci 24:9897-9902.

Huber R, Määttä S, Esser SK, Sarasso S, Ferrarelli F, Watson A, Ferreri F, Peterson MJ, Tononi G (2008) Measures of cortical plasticity after transcranial paired associative stimulation predict changes in electroencephalogram slow-wave activity during subsequent sleep. J Neurosci 28:7911-7918.

Kenet T, Bibitchkov D, Tsodyks M, Grinvald A, Arieli A (2003) Spontaneously emerging cortical representations of visual attributes. Nature 425:954-956.

Lampl I, Reichova I, Ferster D (1999) Synchronous membrane potential fluctuations in neurons of the cat visual cortex. Neuron 22:361-374.

Livy DJ, Wahlsten D (1991) Tests of genetic allelism between four inbred mouse strains with absent corpus callosum. J Hered 82:459-464.

Massimini M, Huber R, Ferrarelli F, Hill S, Tononi G (2004) The sleep slow oscillation as a traveling wave. J Neurosci 24:6862-6870.

Mukhametov LM, Supin AY, Polyakova IG (1977) Interhemispheric asymmetry of the electroencephalographic sleep patterns in dolphins. Brain Res 134:581-584. 
Nir Y, Mukamel R, Dinstein I, Privman E, Harel M, Fisch L, Gelbard-Sagiv H, Kipervasser S, Andelman F, Neufeld MY, Kramer U, Arieli A, Fried I, Malach R (2008) Interhemispheric correlations of slow spontaneous neuronal fluctuations revealed in human sensory cortex. Nat Neurosci 11:1100-1108.

Petersen CC, Hahn TT, Mehta M, Grinvald A, Sakmann B (2003) Interaction of sensory responses with spontaneous depolarization in layer $2 / 3$ barrel cortex. Proc Natl Acad Sci U S A 100:13638-13643.

Poulet JF, Petersen CC (2008) Internal brain state regulates membrane potential synchrony in barrel cortex of behaving mice. Nature 454:881-885.

Prechtl JC, Cohen LB, Pesaran B, Mitra PP, Kleinfeld D (1997) Visual stimuli induce waves of electrical activity in turtle cortex. Proc Natl Acad Sci U S A 94:7621-7626.

Shoham D, Glaser DE, Arieli A, Kenet T, Wijnbergen C, Toledo Y, Hildesheim R, Grinvald A (1999) Imaging cortical dynamics at high spatial and temporal resolution with novel blue voltage-sensitive dyes. Neuron 24:791-802.
Sirota A, Buzsáki G (2005) Interaction between neocortical and hippocampal networks via slow oscillations. Thalamus Relat Syst 3:245-259.

Steriade M, Timofeev I (2003) Neuronal plasticity in thalamocortical networks during sleep and waking oscillations. Neuron 37:563-576.

Steriade M, Nuñez A, Amzica F (1993) A novel slow ( $<1 \mathrm{~Hz}$ ) oscillation of neocortical neurons in vivo: depolarizing and hyperpolarizing components. J Neurosci 13:3252-3265.

Timofeev I, Steriade M (1996) Low-frequency rhythms in the thalamus of intact-cortex and decorticated cats. J Neurophysiol 76:4152-4168.

Timofeev I, Grenier F, Steriade M (2001) Disfacilitation and active inhibition in the neocortex during the natural sleep-wake cycle: an intracellular study. Proc Natl Acad Sci U S A 98:1924-1929.

Waters J, Helmchen F (2006) Background synaptic activity is sparse in neocortex. J Neurosci 26:8267-8277.

Wilson MA, McNaughton BL (1994) Reactivation of hippocampal ensemble memories during sleep. Science 265:676-679. 